\title{
SKULLCAPFLAVONE II INHIBITS PROLIFERATION, MIGRATION, INVASION AND PROMOTES APOPTOSIS OF BREAST CANCER CELLS BY ATTENUATION OF PI3K/AKT/MTOR PATHWAY
}

\author{
LIANRUI LI ${ }^{1}$, ZHIYONG CHEN ${ }^{1}$, JUN YU ${ }^{1}$, and JUN ZHANG ${ }^{2 *}$ \\ 'Department of Pharmacy, Tianjin Beichen District Chinese Medicine Hospital, \\ Tianjin 300400, China \\ ${ }^{2}$ Clinical Pharmacy Office of Pharmacy School of Tianjin Medical University, \\ Tianjin 300070, China
}

\begin{abstract}
Breast cancer is the leading cause of mortality in women across the globe. The study therefore designed to evaluate the effect of Skullcapflavone II (SCP) against breast cancer cells in search of a novel anticancer molecule. The SCP dose selected for the study is 5,10 , and $15 \mu \mathrm{M}$. The effect of SCP on cellular viability and apoptosis of MDA-MB-231 cells was studied by the MTT assay and Annexin V/ PI and DAPI staining, respectively. Results suggested that SCP causes attenuation of viability of MDAMB-231 cells together with damage of cellular morphology and induction of apoptosis in a concentrationdependent manner. In a wound-healing assay and transwell migration assay, SCP causes a reduction in cellular migration and evasion. In a western blot analysis, SCP causes a reduction in Bcl-2 and increases expression of p-53 and Bax together with inhibition of PI3K/Akt/mTOR signaling cascade. Results suggest that SCP inhibits the proliferation and migration of breast cancer cells and may prove beneficial in the management of breast cancer.
\end{abstract}

Keywords: Flavone, proliferation, breast cancer, apoptosis, PI3K

In the majority of the countries of the world, Breast cancer (BC) is the leading cause of cancer-related morbidity and mortality in women. It was classified as a fifth leading cause of death among cancerrelated mortality both in a developed and developing nation $(1,2)$. Moreover, due to the lack of early detection programs, as well as challenges in implementing appropriate diagnostic and treatment approaches, the overall survival of breast cancer patients was found significantly lower in developing nations than in developed nations $(3,4)$. For instance, in China, the incidence of cancer has been raised in the past decade due to rapid economic development and the adoption of the western lifestyle $(5,6)$. Studies suggested that breast cancer is the leading cause of mortality with 16.5 percent of all cancers in females (7). The surgery and chemotherapy is the only viable option in the premature stage of the disease. Therefore, new effective agents are worth to be investigated for the treatment of BC.

Skullcapflavone II (SCP), a flavonoid isolated from $S$. baicalensis, belongs to the class of tetramethoxyflavone. It is substituted by methoxy groups at positions $6,7,8$, and 6' and hydroxy groups at positions 5 and 2' respectively (8). It showed potent inhibition of bradykinin-induced contractions in the rat ileum and uterus (9). It also showed anti-MMP-1 activity, anti-asthmatic and anti-osteoclastogenesis effect (10-14). However, none of the studies has reported the anti-breast cancer potential of SCP, thus, in the present study, we intend to investigate the effect of SCP on breast cancer cells.

\section{EXPERIMENTAL}

\section{Cell line and morphological assessment}

Human gastric cancer BGC823 cells (obtained from Cell Research Institute of the Chinese Academy of Science) were cultured in RPMI-1640 medium supplemented with $10 \% \mathrm{FBS}, 100 \mu \mathrm{g} / \mathrm{mL}$ penicillin, and $100 \mu \mathrm{g} / \mathrm{mL}$ streptomycin in a humidified incubator at $37^{\circ} \mathrm{C}$ with $5 \% \mathrm{CO}_{2}$. SCP (Sigma Aldrich, USA) in serial concentrations as dissolved in DMSO $(5,10$, and $15 \mu \mathrm{M})$ were added to the culture medium. DMSO ( $0 \mu \mathrm{M}$ SCP) was used as a control. After incubation for 24,48 , and $72 \mathrm{~h}$, cell morphological

* Corresponding author: e-mail: junzhang021@yahoo.com 
changes were monitored through an inverted microscope (Zeiss Axio Observer A1).

\section{Cell proliferation assessment using MTT assay}

In brief, the cells were cultured in 96-well flat-bottom microtiter culture plates at a density of $5 \times 10^{4}$ cells per well and subjected to treatment with diverse indicated concentrations of $\operatorname{SCP}(5,10$, and $15 \mu \mathrm{M})$. After 24,48 , and $72 \mathrm{~h}$ of SCP treatment $20 \mu \mathrm{L}$ of a fresh MTT $(2.5 \mathrm{mg} / \mathrm{ml})$ solution was added and were incubated for $4 \mathrm{~h}$ at $37^{\circ} \mathrm{C}$. This was followed by the addition of DMSO to dissolve the formazan. Finally, the absorbance was taken at $570 \mathrm{~nm}$ using a microplate reader.

\section{Assay of cell apoptosis by dual staining with Annexin $V$ and propidium iodide (PI)}

MDA-MB-231 cells along with the varying concentration of $\operatorname{SCP}(5,10$, and $15 \mu \mathrm{M})$ were seeded in well-plates. After $48 \mathrm{~h}$ of incubation, the cells were centrifuged, resuspended in binding buffer, and successively incubated with Annexin V-FITC-PI $(5 \mu \mathrm{l})$ for $15 \mathrm{~min}$ at room temperature. Apoptosis was observed by flow cytometric analysis using a FACSCanto ${ }^{\mathrm{TM}}$ II spectrophotometer (BD Biosciences, San Jose, CA, USA).

\section{DAPI Staining}

Cell nuclear morphology was measured through fluorescence microscopy following DAPI staining. The MDA-MB-321 cells were treated with SCP (5, 10 , and $15 \mu \mathrm{M}$ ) for $24 \mathrm{~h}$. Cells were then washed with PBS, fixed with $4 \%$ formaldehyde, and washed 2 times each for $5 \mathrm{~min}$. Next, cells were stained with DAPI and incubated for $5 \mathrm{~min}$ at room temperature away from light. The cells were finally washed with PBS one time and visualized using a fluorescence microscope (Carl Zeiss, Oberkochen, Germany).

\section{Wound healing assay}

The MDA-MB-321 cells $\left(1 \times 10^{5}\right.$ cells/well $)$ were seeded into 24-well plates and scratched using pipette tips. The detached cells were removed from plates after washing with PBS and then incubated with a complete growth medium containing a diverse concentration of SCP for $24 \mathrm{~h}$. The migration of cells was observed at 0 and 24 h post-induction of injury using a phase-contrast microscope and analyzed using Image $\mathrm{J}$ software (NIH, USA).

\section{Transwell assay}

The various concentration of $\operatorname{SCP}(5,10$, and $15 \mu \mathrm{M})$ was used to treat the breast cancer cells in the upper chamber for $24 \mathrm{~h}$, whereas, DMEM containing
$10 \%$ FBS were added to the bottom chambers. After $24 \mathrm{~h}$ incubation at $37^{\circ} \mathrm{C}$, a cotton swab was used to remove the upper layer of non-migrated cells, whereas, migrated cells on the bottom were stained with crystal violet and visualized under a light microscope after fixing in paraformaldehyde.

\section{Western blot analysis}

Briefly, after the indicated treatment of SCP (5, 10 , and $15 \mu \mathrm{M})$, the cell lysates were collected, and cells were dissolved in lysis buffer. The cell lysate, obtained after sonication and boiling, was loaded to $10 \%$ SDS-PAGE, separated by electrophoresis, and then transferred to a PVDF film. The film was then incubated with corresponding primary antibody followed by HRP-conjugated secondary antibody after being blocked (TBS containing $0.1 \%$ Tween-20, and $5 \%$ de-fat milk), The protein lanes were visualized using ECL Western Blotting Detection Kit (GE Healthcare, USA).

\section{Statistical analysis}

All the data were expressed as mean \pm standard error (SEM). Statistical analysis was performed using one-way analysis of variance (ANOVA) followed by Bonferroni post hoc multiple comparison test using statistical software GraphPad Prism 5.0 (California, USA). The P-value $<0.05$ was considered statistically significant.

\section{RESULTS}

\section{Effect of SCP on cellular viability and morphology of MDA-MB-231 cells}

The effect of SCP was evaluated on the viability of human breast cancer cells (MDA-MB-231) via MTT assay. As presented in fig. 1, it has been found that SCP causes significant attenuation of cellular viability of MDA-MB-231 cells. The most significant inhibition was observed at the highest test dose of $15 \mu \mathrm{M}(* * * \mathrm{P}<0.001 v s$ the control group) at $72 \mathrm{~h}$. Moreover, SCP also alters the integrity of the cellular architecture of MDA-MB-231 cells in the microscopic image as revealed in Figure 1B.

\section{Effect of SCP on apoptosis of MDA-MB-231 cells}

To confirm whether the effects of SCP on the cytotoxicity of MDA-MB-321 cells were related to apoptosis, an Annexin V assay was performed. As shown in Fig 2A, SCP dose-dependently increased apoptosis in MDA-MB-321 cells. The cells were stained with DAPI to better represent the obvious morphological changes related to apoptosis and 


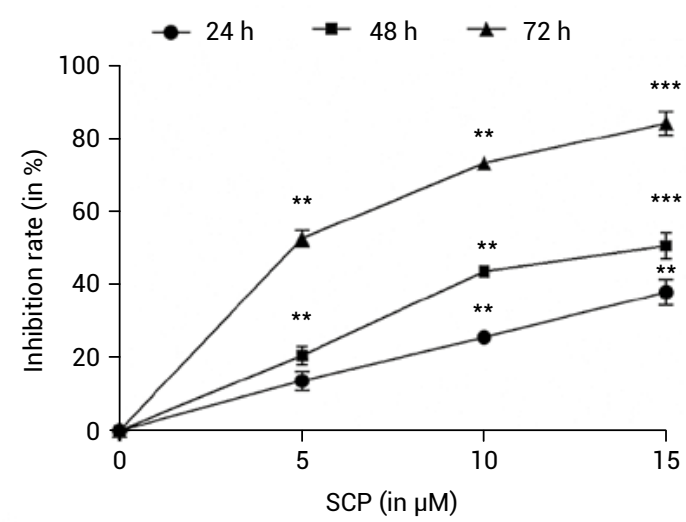

Figure 1. Effect of SCP on (A) cellular viability on MDA-MB-231 cells and (B) cellular integrity. Results represent means \pm SEM of three independent experiments. ${ }^{* *} P<0.01$; $* * * P<0.001$ vs the control group.

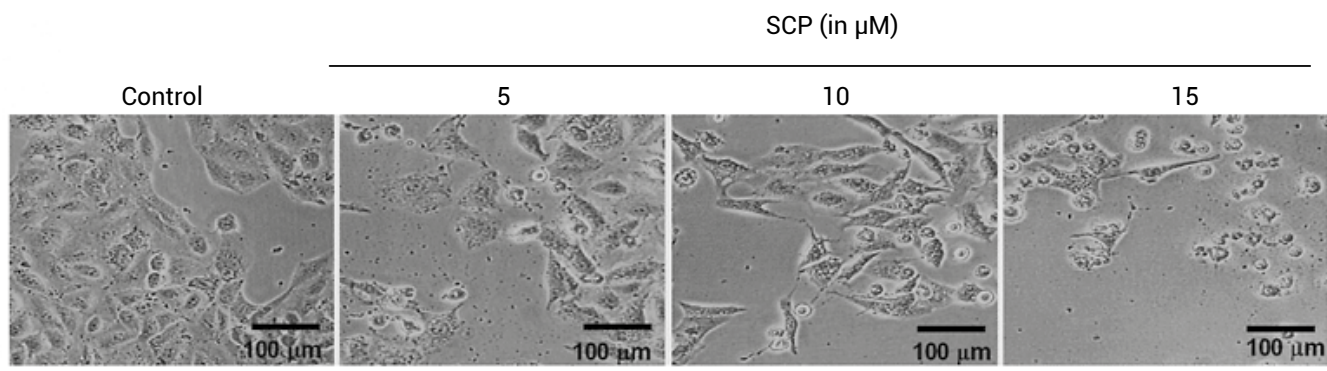

results have been shown in Figure 2B. These results indicated that SCP induced cytotoxicity by induction of apoptosis.

\section{Effect SCP on the migration of MDA-MB-231 cells}

The next part of the study was aimed to explore the effect of SCP on the migration of MDAMB-231 cells by scratch test and Transwell invasion assay. It has been found that SCP causes a reduction in the migration of cells around the wound corner, Fig 3A. It has been found that the rate of sclerosis on both sides is decreased with an increase in sclerosis width. Moreover, the scratch-healing rate of the SCP-treated group was found reduced in a dosedependent manner (Figure 3A). The SCP-treated group also showed a reduction in the invasion ability of MDA-MB-231 cells. These results indicated that SCP possesses the ability to attenuate tumor metastasis.

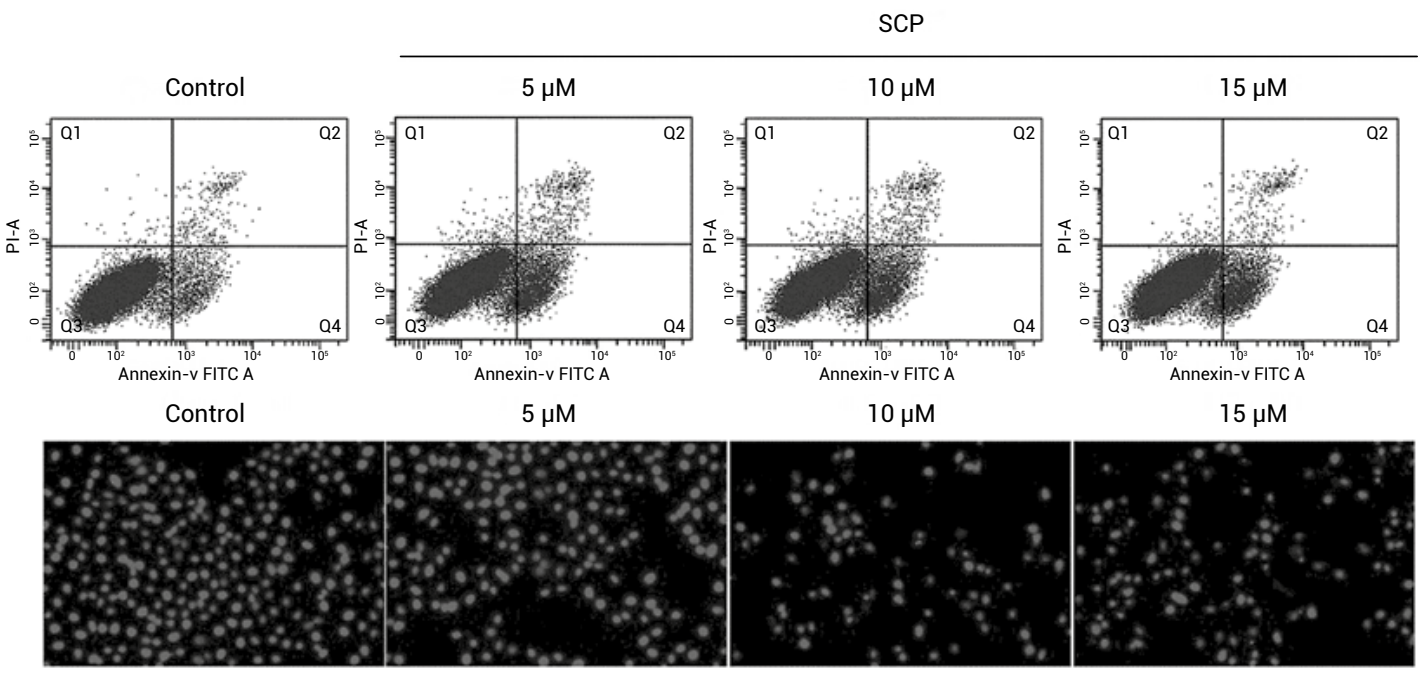

Figure 2. Effect of SCP on (A) apoptosis by Annexin-PI staining, and (B) cellular morphology by DAPI assay in MDA-MB-231 cells. 


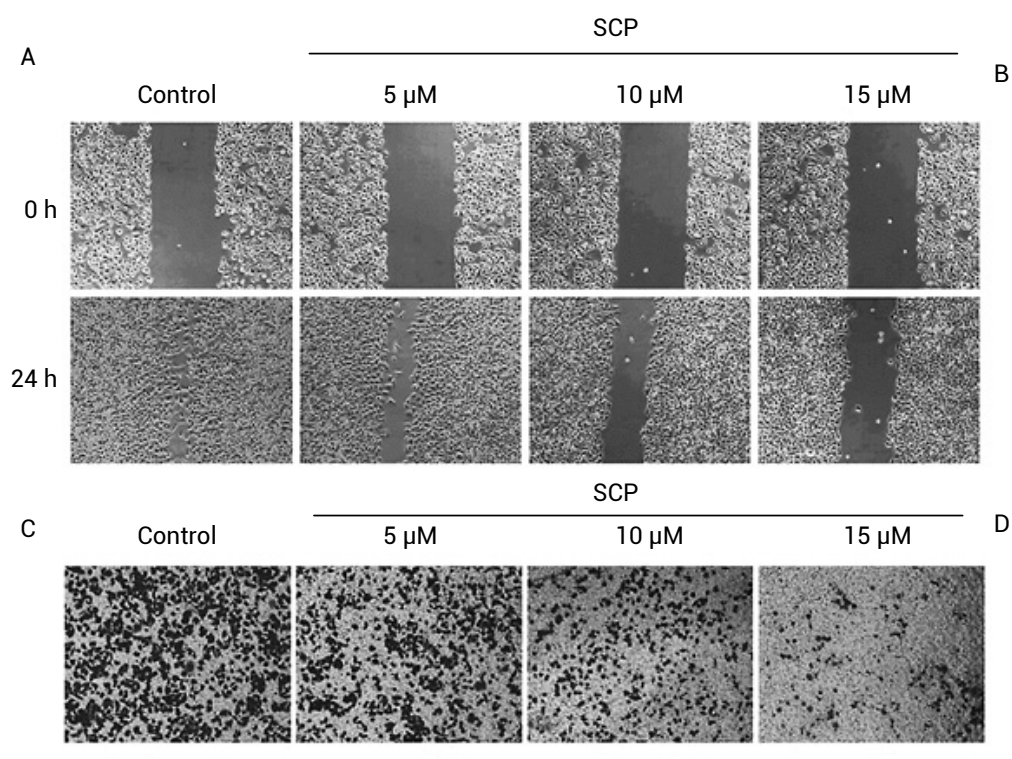

Figure 3. Effect of SCP on the cellular migration of MDA-MB-231 cells in wound healing assay (A and B) transwell migration assay $(\mathrm{C}$ and $\mathrm{D})$. Results represent means $\pm \mathrm{SEM}$ of three independent experiments. $* * \mathrm{P}<0.01 v s$ the control group.

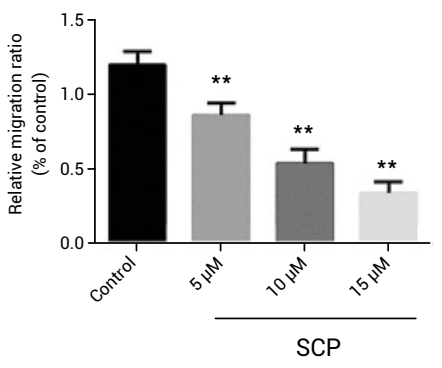

\section{Effect of SCP on the mitochondria-associated apoptotic protein}

Western blot analysis was performed to determine the effect of SCP on the expression of mitochondria-associated intrinsic apoptosis protein. As shown in Figure 4, the SCP-treated group showed an increase in the expression of p-53 and Bax, while the level of $\mathrm{Bcl} 2$ was found to decrease significantly. The highest tested dose of SCP $\left(15 \mu \mathrm{M},{ }^{*} \mathrm{P}<0.01\right)$ causes a more significant effect than other tested doses which suggests that SCP causes dose-dependent reduction of the mitochondria-associated apoptotic proteins.

\section{Effect of SCP on the PI3K/Akt/mTOR signaling cascade}

The effect of SCP on the PI3K/Akt/mTOR downstream signaling proteins was analyzed by using western blot analysis and results are presented in Figure 5. It has been found that the level p-PI3K, p-Akt, and p-mTOR was declined significantly in SCP treated group as compared to the control, while

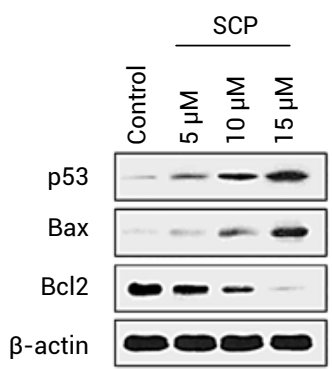

B

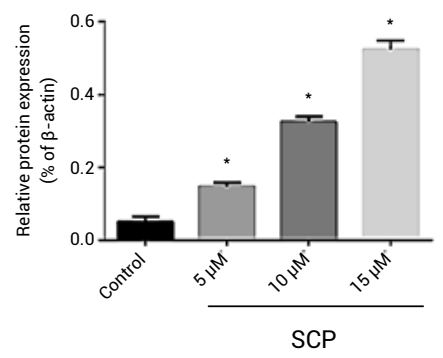

Figure 4. Effect of SCP on the mitochondria-associated apoptotic proteins as determined by western blot in MDA-MB-231 cells (A). Representative graph of p-53 (B), Bax (C) and Bcl2 (D). Results represent means \pm SEM of three independent experiments. ${ }^{*} \mathrm{P}<0.01$ vs the control group.
C

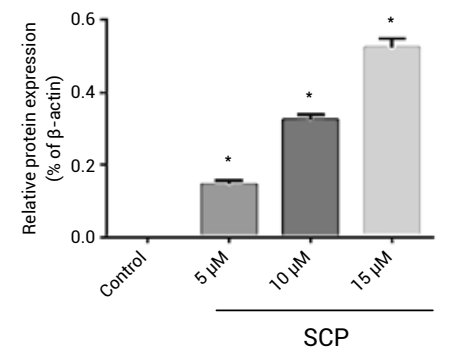

D

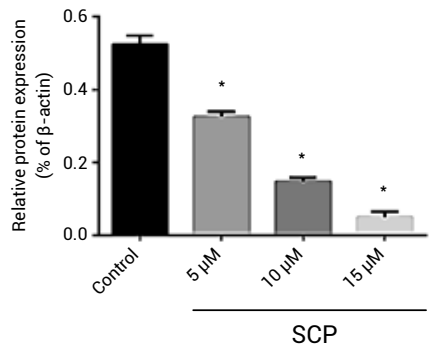


A

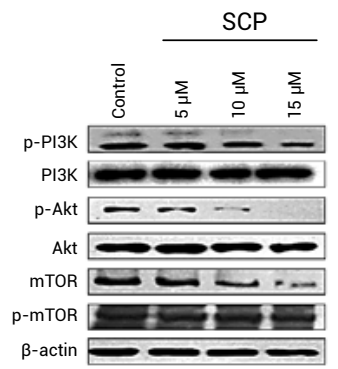

B

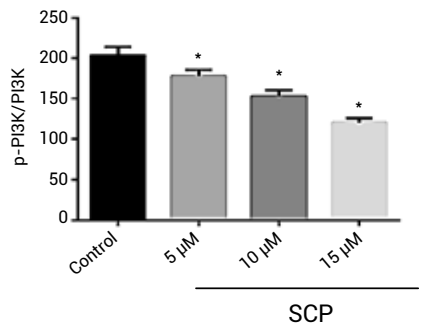

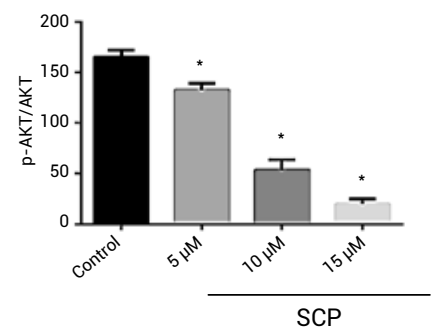

Figure 5. Effect of SCP on the (A) PI3K/Akt $/$ mTOR pathway in MDA-MB-231 as shown by western blot analysis, representative densitometry graph of (B) p-PI3K, (C) p-Akt, and (D) mTOR. Results represent means \pm SEM of three independent experiments. $* \mathrm{P}<0.01$ vs the control group. no significant changes were observed in the nonphosphorylated counterparts. Thus, it could be understood that SCP might exert an anticancer effect against breast cancer cells by inhibition of the PI3K/ Akt/mTOR downstream signaling cascade.

\section{DISCUSSION}

Despite significant advances in the diagnostic and therapeutic modalities against breast cancer, none of the agents or strategies were found successful and that made breast cancer the leading cause of death among women $(15,16)$. However, till now, no reports have been published which discuss the effect of Skullcapflavone II (SCP) in breast cancer. Therefore, in the present study, we have shown the protective effect of SCP on breast cancer cells and its possible mechanism of action. In previous studies, SCP showed strong bradykinin antagonistic activity with potent anti-inflammatory activity in an animal model of asthma $(9,12)$.

In the present study, SCP causes significant attenuation of viability of MDA-MB-231 cells as determined by the MTT assay. This could be easily understood by the fact that, the cells treated with SCP showed loss of cellular integrity and architecture upon microscopic examination and which might be the reason for the death of cancer cells. To understand the molecular mechanism behind the anti-proliferative effect of SCP, we have performed series of experiments. Therefore, in the next part, we examined the role of SCP on apoptosis. Apoptosis is the process termed programmed cell death by which the body will try to eliminate the dysfunctional cells (17). In cancer, the cancerous cells evade this process and make it dysfunctional which leads to their survival. The process of apoptosis is highly governed by pro-apoptotic and apoptotic genes, and oncogenic mutation of these genes disrupts apoptosis, leading to tumor survival and metastasis $(18,19)$. Many studies have confirmed the role of many cytotoxic agents in promoting apoptosis (20). In the present study, SCP promotes apoptosis as shown in fluorescence microscopy of DAPI stained cells. The cancer treatment has faced serious obstacles from the metastasis of tumors which was initiated by the migration and invasion process (21-23). Thus, the current approaches to control cancer cell metastasis have been concentrated on reducing the migration and invasion process. A recent study revealed that SCP causes inhibition of migration and invasion in MDA-MB-231 cells. The next part of the study was concentrated on studying the effect of SCP on the Bcl2 family proteins. These proteins are considered proapoptotic and anti-apoptotic which govern cellular proliferation. It has been found that the expression of p-53 and Bax, while the level of Bcl2 was found to be decreased significantly in SCP treated group (24-26). $\mathrm{PI} 3 \mathrm{~K} / \mathrm{Akt} / \mathrm{mTOR}$ is a signal transduction pathway, which is directly linked to the growth and development of diverse cancers. It has been found that activation of the PI3K/Akt/mTOR signaling pathway plays an important role in cellular proliferation, apoptosis, and metastasis and is found aberrantly activated in many cancers including breast (27). Therefore, many of the newer anticancer drugs inhibit this signaling pathway, such as Duvelisib, Copanlisib, and Idelalisib (28). Recently, Alpelisib an alpha-specific PI3K inhibitor was approved by the FDA in May 2019 for use in 
combination with endocrine therapy fulvestrant for the treatment of HR-positive and HER2/neu-negative breast cancer $(29,30)$. In the present study, SCP causes inhibition of the expression of phosphorylated proteins involved in the signaling cascade of PI3K/AKT/ mTOR revealing the possible mechanism behind SCPinduced anti-breast cancer effects.

This present study has some limitations because we have used only one breast cancer cell (MDAMB-231) and other cell lines need to be investigated in the future to support these results. Our study also lacks an in-vivo study to support our claim, which we may consider in our future experiments.

\section{CONCLUSION}

In summary, we demonstrated that Skullcapflavone II induced apoptosis and inhibit migration and evasion of breast cancer cells through attenuating the phosphorylation of PI3K, AKT, and mTOR. These findings improved the understanding of the anti-breast cancer activities of Skullcapflavone II.

\section{Acknowledgments}

Authors thanks to the Pharmacy School of Tianjin Medical University.

\section{Conflict of interest}

The authors declare no conflicts of interest.

\section{REFERENCES}

1. Ferlay J., Shin H.R., Bray F., Forman D., Mathers C., Parkin D.M.: Int. J. Cancer 127, 2893 (2010).

2. Torre L.A., Bray F., Siegel R.L., Ferlay J., LortetTieulent J., Jemal A.: CA Cancer J. Clin. 65, 87 (2015).

3. Shukla N., Hagenbuchner M., Win K.T., Yang J.: Comput. Methods Programs Biomed. 155, 199 (2018).

4. Bray F., Ferlay J., Soerjomataram I., Siegel R.L., Torre L.A., Jemal A.: CA Cancer J Clin. 68, 394 (2018).

5. Bray F., Ferlay J., Soerjomataram I., Siegel R.L., Torre L.A., Jemal A.: CA Cancer J Clin. 68, 394 (2018).

6. Feng R.M., Zong Y.N., Cao S.M., Xu R.H. Cancer Commun. (Lond), 39, 22 (2019).
7. Fan L., Strasser-Weippl K., Li J.J., St Louis J., Finkelstein D.M., Yu K.D, et al.: Lancet Oncol. 15, e729 (2014).

8. Kubo M., Matsuda H., Tani T., Arichi S., Kimura Y., Okuda H.: Chem. Pharm. Bull. 33, 2411 (1985).

9. Yun-Choi H.S., Chung S.H., Kim Y.J.: Arch. Pharm. Res. 16, 283 (1993).

10. Lee Y.H., Seo E.K., Lee S.T.: Int. J. Mol. Sci. 20, 11 (2019).

11. Bui T.T., Piao C.H., Song C.H., Chai O.H.: Int. Immunopharmacol. 52, 77 (2017).

12. Jang H.Y., Ahn K.S., Park M.J., Kwon O.K., Lee H.K., Oh SR.: Int Immunopharmacol. 12, 666 (2012).

13. Lee J., Saem Son H., In Lee H., Lee G.R., Jo Y.J., et al.: FASEB J. 33, 2026 (2019).

14. Lee S., Lee Y.: J. Invest. Dermatol. 139, S327 (2019).

15. Hutchinson L.: Nat. Rev. Clin. Oncol. 7, 669 (2010).

16. Sledge G.W., Mamounas E.P., Hortobagyi G.N., Burstein H.J., Goodwin P.J., Wolff A.C.: J. Clin. Oncol. 32, 1979 (2014).

17. Elmore S.: Toxicol. Pathol. 7, 495 (2007).

18. Tsujimoto Y., Shimizu S.: Apoptosis 12, 835 (2007).

19. Lin Q.S.: Acta Biochim. Biophys. Sin. (Shanghai) 31, 118 (1999).

20. Hunter A.M., LaCasse E.C., Korneluk R.G. Apoptosis 12, 1543 (2007).

21. Chaffer C.L., Weinberg R.A.: Science 331, 1559 (2011).

22. Gupta G.P., Massagué J.: Cell 17, 679 (2006).

23. Steeg P.S.: Nat. Rev. Cancer 16, 201 (2016).

24. Yip K.W., Reed J.C.: Oncogene 27, 6398 (2008).

25. Ashkenazi A., Fairbrother W.J., Leverson J.D., Souers A.J.: Nat. Rev. Drug Discov. 16, 273 (2017).

26. Delbridge A.R.D., Grabow S., Strasser A., Vaux D.L.: Nat. Rev. Cancer 16, 99 (2016).

27. Lee J.J.X., Loh K., Yap Y.S.: Cancer Biol. Med. 12, 342 (2015).

28. Massacesi C., di Tomaso E., Urban P., Germa C., Quadt C., Trandafir L.: OncoTargets Ther. 9, 203 (2016).

29. André F., Ciruelos E., Rubovszky G., Campone M., Loibl S., Rugo H.S.: N. Engl. J. Med. 380, 1929 (2019).

30. Mayer I.A., Abramson V.G., Formisano L., Balko J.M., Estrada M. V., Sanders M.E., Clin Cancer Res. 23, 26 (2017). 